\title{
Análisis bibliométrico de la producción científica internacional sobre biomarcadores salivales durante el período 2004-2018
}

Bibliometric analysis of international scientific production on salivary biomarkers during the period 2004-2018

\author{
Análise bibliométrica da produção científica internacional em \\ biomarcadores salivares no período 2004-2018
}

\section{Rolando Pablo Alejandro Juárez ${ }^{1}$}

\begin{abstract}
Recibido: 10 de febrero de 2020
Aprobado: 16 de diciembre de 2020

Publicado: 31 de diciembre de 2020

Cómo citar este artículo: Juárez RPA. Análisis bibliométrico de la producción científica internacional sobre biomarcadores salivales durante el período 2004-2018. Revista Nacional de Odontología. (2020); 16(2), 1-16. doi: https://doi.org/10.16925/2357-4607.2020.02.08
\end{abstract}

Artículo de investigación. https://doi.org/10.16925/2357-4607.2020.02.08

1 Doctor en Odontología. Universidad Nacional del Nordeste. Facultad de Odontología. Corrientes. Argentina.

Correo electrónico: ropablojuarez@odn.unne.edu.ar ORCID: https://orcid.org/0000-0001-8950-3373 
Análisis bibliométrico de la producción científica internacional sobre biomarcadores salivales durante el período 2004-2018

\section{Resumen}

Introducción: la saliva protege la integridad de los tejidos orales, pero también ofrece evidencias de enfermedades locales y sistémicas. La bibliometría en estudios relacionados con la odontología es poco común en la literatura. Desde esta perspectiva, el objetivo de este estudio fue realizar el mapeo del panorama científico relacionado con la investigación de biomarcadores salivales en todo el mundo entre los años 2004 y 2018.

Métodos: este estudio adoptó un método bibliométrico. Se realizó una investigación documental, observacional, descriptiva, retrospectiva, con descripción cuantitativa de los datos. Dentistry \& Oral Sciences Source ha sido seleccionada como base de datos bibliométrica en línea para buscar y recuperar documentos. Todos los datos presentados en este estudio se analizaron utilizando los siguientes programas de software: VOSviewer, Publish or Perish, Mendeley Desktop y SPSS versión 19 para Windows. Se utilizaron, métodos estadísticos descriptivos e inferenciales.

Resultados: se registraron 218 documentos. Las medias y desviación estándar de las publicaciones entre 2004-2011 $(4,67 \pm 1,52)$ y 2012-2018 $(24,67 \pm 2,47)$ mostraron diferencias significativas $(p=0.001)$. La enfermedad periodontal (37\%) y el cáncer oral (20\%) fueron los temas de diagnóstico más estudiados. Wong DT (Universidad de California en Los Ángeles, EE. UU.) fue el autor con más artículos (5,96\%).

Conclusión: durante el período de estudio, se incrementó la investigación internacional sobre biomarcadores salivales, lo que indica la importancia de los componentes salivales para los enfoques novedosos de pronóstico, diagnóstico y monitoreo de pacientes con enfermedades orales y sistémicas.

Palabras clave: saliva, biomarcadores, bibliometría, publicaciones.

\section{Abstract}

Introduction: saliva protects the integrity of oral tissues, but also offers evidence of local and systemic diseases. Bibliometry in studies related to dentistry is uncommon in the literature. From this perspective, the objective of this study was to map the scientific landscape related to the research of salivary biomarkers around the world between 2004 and 2018.

Methods: This study adopted a bibliometric method. A documentary, observational, descriptive, retrospective research was conducted, with quantitative description of the data. Dentistry \& Oral Sciences Source has been selected as an online bibliometric database to search and retrieve documents. All data presented in this study were analyzed using the following software programs: VOSviewer, Publish or Perish, Mendeley Desktop and SPSS version 19 for Windows. Descriptive and inferential statistical methods were used.

Results: 218 documents were registered. The means and standard deviation of the publications between 2004-2011 (4.67 \pm 1.52$)$ and 2012-2018 $(24.67 \pm 2.47)$ showed significant differences $(p=0.001)$. Periodontal disease $(37 \%)$ and oral cancer $(20 \%)$ were the most studied diagnostic subjects. Wong DT (University of California, Los Angeles, USA) was the author with the most articles (5.96\%).

Conclusion: during the study period, international research on salivary biomarkers was increased, indicating the importance of salivary components for novel approaches to prognosis, diagnosis and monitoring of patients with oral and systemic diseases.

Keywords: saliva, biomarkers, bibliometrics, publications. 


\section{Resumo}

Introdução: a saliva protege a integridade dos tecidos orais, mas também oferece evidências de doenças locais e sistêmicas. Bibliometria em estudos relacionados à odontologia é incomum na literatura. Nessa perspectiva, o objetivo deste estudo foi mapear o cenário científico relacionado à pesquisa de biomarcadores salivares em todo o mundo entre 2004 e 2018.

Métodos: Este estudo adotou um método bibliométrico. Foi realizada pesquisa documental, observacional, descritiva e retrospectiva, com descrição quantitativa dos dados. Dentistry \& Oral Sciences Source foi selecionada como um banco de dados bibliométrico on-line para pesquisar e recuperar documentos. Todos os dados apresentados neste estudo foram analisados utilizando os seguintes programas de software: VOSviewer, Publish or Perish, Mendeley Desktop e SPSS versão 19 para Windows. Métodos estatísticos descritivos e inferenciais foram utilizados.

Resultados: 218 documentos foram registrados. As médias e desvio padrão das publicações entre 2004$2011(4,67 \pm 1,52)$ e 2012-2018 (24,67 $\pm 2,47)$ apresentaram diferenças significativas $(p=0,001)$. Doença periodontal $(37 \%)$ e câncer de boca $(20 \%)$ foram os assuntos diagnósticos mais estudados. Wong DT (Universidade da Califórnia, Los Angeles, EUA) foi o autor com mais artigos (5,96\%).

Conclusão: durante o período do estudo, aumentou a pesquisa internacional sobre biomarcadores salivares, indicando a importância dos componentes salivares para novas abordagens de prognóstico, diagnóstico e monitoramento de pacientes com doenças bucais e sistêmicas.

Palavras-chave: saliva, biomarcadores, bibliometria, publ

\section{Introducción}

La saliva es un líquido corporal secretado por glándulas salivales con propiedades físicas versátiles. Esencialmente contiene agua (99\%), proteínas y electrolitos. Los componentes forman una solución viscoelástica que mantiene la homeostasia de la cavidad oral (1).

El avance de la tecnología ha permitido reconocer la saliva como un fluido para el diagnóstico de enfermedades. Es un procedimiento no invasivo, con recolección segura, económica y fácil de realizar (2). Se necesitan muestras de baja cantidad para la detección de los analitos y existe correlación entre los biomarcadores salivales y plasmáticos (3).

El término, biomarcador, se refiere a parámetros bilógicos medibles y cuantificables que pueden servir como indicadores para la salud y evaluaciones fisiológicas, como los procesos patógenos, exposición ambiental, diagnóstico y pronóstico de enfermedades o respuestas farmacológicas a una intervención terapéutica (4).

El desarrollo de pruebas de laboratorio para biomarcadores salivales, altamente sensibles y precisas para la detección temprana de enfermedades, convirtió a la saliva en un fluido diagnóstico valioso (5). Reflejando esta importancia, la investigación sobre saliva ha aumentado notablemente en los últimos años. Un mejor conocimiento 
de los componentes bioquímicos se ha logrado a través de genómica, proteómica y peptidómica, transcriptómica, metabolómica y microbioma de la saliva humana (6).

Por lo tanto, es esencial examinar sistemáticamente la literatura sobre biomarcadores salivales, para proporcionar una comprensión profunda sobre el último desarrollo del tema. En este sentido, la bibliometría es un conjunto de técnicas reconocidas para el análisis cuantitativo sistemático de documentos de un campo específico de investigación (7).

Así, la revisión de la literatura mediante el análisis bibliométrico se ha utilizado ampliamente para mostrar el desarrollo de la investigación en una disciplina determinada de las ciencias de la salud, como diabetes y oncología. (8, 9). En odontología, este tipo de análisis se ha realizado en diferentes áreas de estudio como implantes, caries y ortodoncia (10-12).

El objeto de estudio de la bibliometría son las características micro y macro de los sistemas de documentos (13). Identifica las tendencias de investigación y los problemas comunitarios. Así, desempeña un papel fundamental en la formulación de políticas públicas y futuras investigaciones sobre la práctica clínica (14).

Como la bibliometría trabaja con metadatos científicos, se utiliza el mapeo bibliométrico que permite interpretar grandes volúmenes de datos mediante la visualización interactiva de imágenes de redes bibliométricas que representan patrones significativos del tema en estudio (15).

El objetivo de este trabajo es describir la producción científica internacional escrita sobre biomarcadores salivales y generar una línea de investigación documental que sirva como insumo de base para la gestión de políticas públicas.

\section{Materiales y métodos}

Se realizó una investigación documental, estudio observacional, descriptivo y retrospectivo. Mediante método bibliométrico se analizó cuantitativamente las publicaciones de autores internacionales sobre biomarcadores salivales.

La búsqueda fue realizada en la base de datos Dentistry \& Oral Sciences Source/EBSCOhost (DOSS), elegida para la investigación por poseer todas las facetas relacionadas con las áreas de odontología (16).

La búsqueda se realizó durante el mes de diciembre 2018, con una revisión semanal hasta el mes de junio 2019, para evitar sesgos introducidos por la renovación rápida de la base de datos. Durante el periodo 2019/01/01-2019/06/31 se realizó una revisión manual de los resúmenes de cada uno de los documentos recuperados. 
Para la pesquisa, inglés fue el idioma seleccionado en la interfaz del host de EBSCO. Se realizó primero una búsqueda básica, mediante los términos "salivary biomarkers" y como limitador "fechas de publicación: 2004/01/01-2018/12/31".

No se realizó un recorte documental, se incluyeron todos los tipos de trabajos encontrados por ser relevantes para el tema de investigación. Si el investigador consideraba que la información proporcionada por la publicación en formato "abstract" era insuficiente para asignar cualquiera de las variables, se revisaba el texto completo de la publicación.

Se utilizaron "limitadores" para acotar la búsqueda: tipo de artículo (ensayos clínicos aleatorizados, metaanálisis, revisiones sistemáticas, revisiones narrativas, casos y controles, estudios observacionales, estudios multicéntricos), idioma, temas (diferentes tópicos identificados en materia: diagnóstico, técnicas de análisis, enfermedades), categorías de revistas (listado de revistas con el número de publicaciones).

Mediante búsqueda avanzada, se realizaron las combinaciones lógicas con el operador booleano AND (campo: autor, términos temáticos, nombre de la publicación, términos geográficos, palabras clave). Así, para determinar la contribución de cada país a la investigación de la saliva, se incluyó una lista completa de los países al término específico "salivary biomarkers".

Los temas se seleccionaron y agruparon de acuerdo a la frecuencia de las palabras clave y la utilización de operadores booleanos.

Se desarrolló un formulario electrónico de extracción de datos con Microsoft Excel 2010 (Redmond, Washington, EE. UU.). Las variables que se incluyeron en el formulario fueron las aportadas por los limitadores de la base de datos. El gestor de referencias bibliográficas Mendeley Desktop versión 1.19.4-win32 (Mendeley Ltd., Elsevier), permitió almacenar, organizar y gestionar los documentos. El software Publish or Perish 6.0 (Harzing, Middlesex University, London) se usó para determinar el número promedio de autores por artículo. El software VOSviewer versión 1.6.11 (Universidad de Leiden, Países Bajos), se utilizó para construir mapas y grupos de redes de colaboración (autores y palabras clave) y poder determinar la fuerza de enlace total (FET).

Para la validación de la estrategia de búsqueda se utilizó el enfoque de Sweileh (17) modificado parcialmente. Consistió en la ausencia de resultados falsos positivos en los 200 principales documentos encontrados y la ausencia de resultados falsos negativos al comparar los datos obtenidos con los programas de software con los proporcionados por la búsqueda manual.

Las variables de estudio fueron: extensión bibliométrica (total de artículos), crecimiento de la documentación, productividad de los autores, análisis de citación, 
colaboración científica, idiomas de publicaciones, tema (asunto, materia), categorías de documentos, factor de impacto de las revistas, productividad por estado nacional.

Todos los datos presentados en este estudio se analizaron utilizando SPSS versión 19 para Windows, mediante métodos estadísticos descriptivos e inferenciales.

\section{Resultados}

El total de las publicaciones encontradas en el periodo 2004-2018, fue de 218, representando el 3,51\% del total de las publicaciones en relación con la saliva (6194).

Los primeros estudios se observan en el año 2004, con un crecimiento irregular a partir del año 2009 (véase figura 1).

Las medias y desviación estándar de las publicaciones entre 2004-2011 (4,67 $\pm 1,52)$ y entre $2012-2018(24,67 \pm 2,47)$ mostraron diferencias significativas ( $p=0.001$, prueba T).

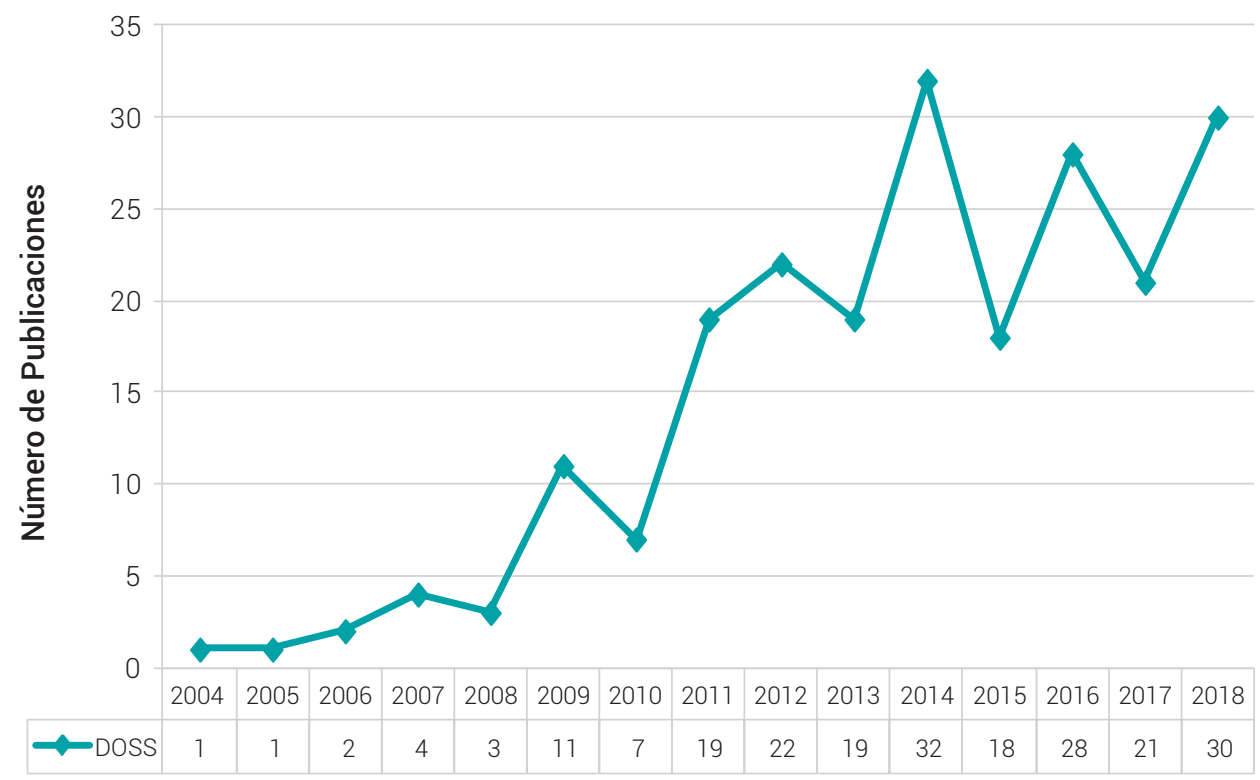

Figura 1. Distribución anual de las publicaciones en la base de datos Fuente: elaboración propia

De los 996 autores totales, Wong fue el autor más productivo (5,96\% del total de trabajos, 2 veces como autor principal y 11 como coautor) y Sorsa presentó la mayor FET (véase tabla 1). 
Tabla 1. Autores más productivos en DOSS

\begin{tabular}{lccclc}
\hline \multicolumn{1}{c}{ Autores } & NP & FET & AC & \multicolumn{1}{c}{ Institución. País } & Temática \\
\hline David T Wong & 13 & 48 & 8,5 & Universidad de California, EE. UU. & $\begin{array}{c}\text { CO, genómica, } \\
\text { proteómica }\end{array}$ \\
\hline Jeffrey L Ebersole & 10 & 67 & 8,8 & Universidad de Kentucky, EE. UU. & EP \\
\hline Craig S Miller & 9 & 64 & 8,8 & Universidad de Kentucky, EE. UU. & EP \\
\hline Timo Sorsa & 8 & 76 & 9,4 & $\begin{array}{l}\text { Universidad de Helsinki, Finlandia. Instituto } \\
\text { Karolinska, Suecia. }\end{array}$ & EP, MMPS \\
\hline Giannobile William V & 8 & 58 & 19,4 & Universidad de Michigan, EE. UU. & EP \\
\hline Richard J Kryscio & 8 & 55 & 9,5 & Universidad de Kentucky, EE. UU. & EP \\
\hline Taina Tervahartiala & 6 & 59 & 7,7 & Universidad de Helsinki, Finlandia. & EP, MMPS \\
\hline Terry Rees & 5 & 34 & 4 & Universidad de Texas, EE. UU. & CO \\
\hline
\end{tabular}

$\mathrm{NP}=$ número de publicaciones, FET = fuerza de enlace total, $\mathrm{AC}=$ análisis de citas (promedio de citas por publicación), $\mathrm{EP}=$ enfermedad periodontal, $\mathrm{MMPs}=$ metaloproteinasas de matriz, $\mathrm{CO}=$ cancer oral

Fuente: elaboración propia

Las redes principales de coautoría identificadas entre los autores más productivos, fueron: 1) 60 autores (70\% de instituciones finlandesas y 61,67\% suecas), de la Universidad de Helsinki (investigador de referencia [IR]: Sorsa y Tervahartiala) en un 66,67\% (véase figura 2) y 50\% del Instituto Karolinska (IR: Sorsa); 2) 46 autores (91,30\% de instituciones americanas, EE. UU.), 67,39\% de la Universidad de Michigan (IR: Giannobile); 3) 44 autores (77,27\% de instituciones americanas, EE. UU.), 31,81\% de la Universidad de California (IR: Wong); 4) 30 autores (100\% de instituciones americanas, EE. UU.), 80\% de la Universidad de Kentucky (IR: Ebersole, Miller y Kryscio); 5) 15 autores (93,33\% de instituciones americanas, EE. UU.), 60\% de la Universidad de Texas (IR: Rees). 
8 Análisis bibliométrico de la producción científica internacional sobre biomarcadores salivales durante el período 2004-2018

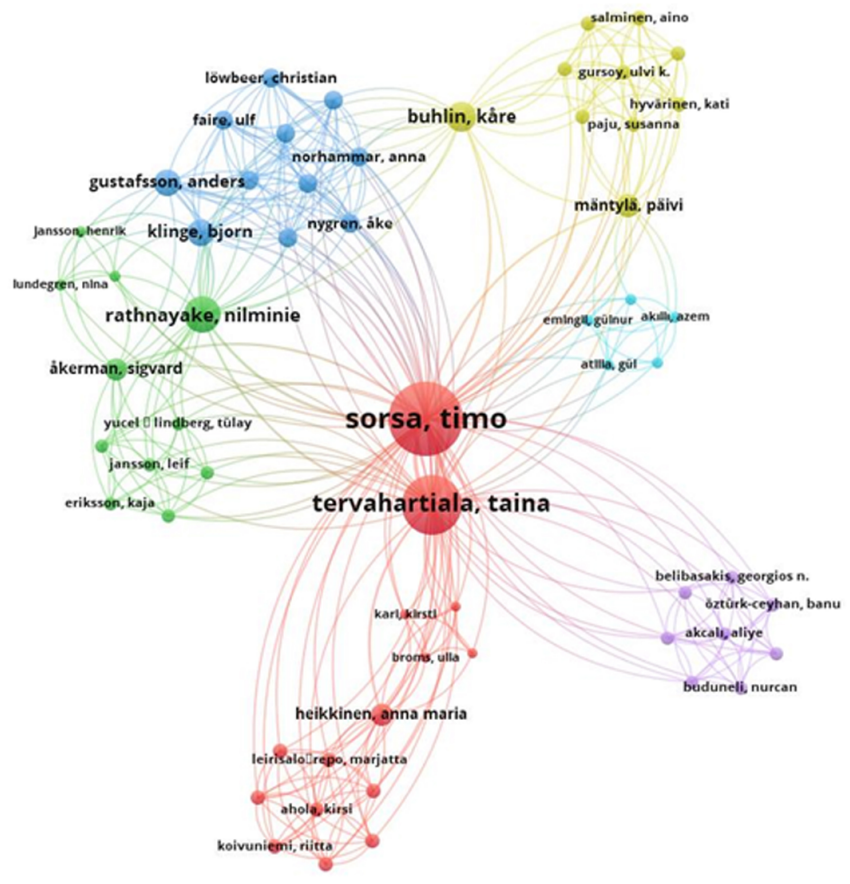

\& vosviewer

Figura 2. Redes de colaboración de autores europeos

Fuente: elaboración propia

El número de autores por trabajo se incrementó durante el periodo de estudio; el promedio entre 2004-2011 fue de 4,42 y entre 2012-2018 de 5,12. Sobre la base del índice de Lotka, predominaron los pequeños productores (con 1 trabajo) en un 90,96\%; mientras que los medianos productores (entre 2 y 9 trabajos) fueron el $8.83 \%$ y los grandes productores (10 o más trabajos) el 0,20\%. Seis de los ocho autores más productivos fueron de USA.

Los temas de diagnóstico más abordados estuvieron asociados con la enfermedad periodontal (37\%) y el cáncer oral (20\%). Las temáticas de las revistas con 10 o más publicaciones sobre biomarcadores salivales, en el periodo de estudio, guardaron correspondencia con los temas predominantes (véase tabla 2). Prevaleció el idioma inglés en todas las instancias de análisis con un 98,16\%. 
Tabla 2. Revistas con mayor producción en DOSS

\begin{tabular}{|c|c|c|c|c|c|}
\hline Revistas & NP & FI-2004 & FI-2018 & País & Temática \\
\hline Journal of Periodontology & 20 & 1.904 & 2.605 & EE.UU. & Periodoncia \\
\hline $\begin{array}{l}\text { Journal of Clinical Perio- } \\
\text { dontology }\end{array}$ & 14 & 2.081 & 4.184 & Reino Unido & Periodoncia e implantología \\
\hline Oral Diseases & 13 & 1.676 & 2.481 & Reino Unido & Ciencias orales \\
\hline $\begin{array}{l}\text { Journal of Oral Pathology } \\
\text { \& Medicine }\end{array}$ & 11 & 1.818 & 2.125 & Reino Unido & Patología y medicina oral \\
\hline Oral Oncology & 11 & 2.386 & 3.893 & Reino Unido & $\begin{array}{l}\text { Neoplasias en la cabeza y el } \\
\text { cuello }\end{array}$ \\
\hline Archives of Oral Biology & 10 & 1.262 & 1.793 & Reino Unido & $\begin{array}{l}\text { Ciencias orales y craneofa- } \\
\text { ciales }\end{array}$ \\
\hline $\begin{array}{l}\text { Clinical Oral Investiga- } \\
\text { tions }\end{array}$ & 10 & 0.901 & 2.587 & Alemania & $\begin{array}{l}\text { Ciencia y medicina oral y } \\
\text { maxilofacial }\end{array}$ \\
\hline
\end{tabular}

$\mathrm{NP}=$ número de publicaciones, $\mathrm{Fl}=$ factor de impacto (periodo de dos años)

Fuente: elaboración propia

En el registro de palabras claves de frecuencia (F) alta, de 1011 palabras se analizaron 90 con una F mínima de 5. Las asociadas a enfermedad periodontal (9 palabras) presentaron una $F$ total de 125 y una FET de 832. Las relacionadas con cáncer (9 palabras) presentaron una F total de 88 y una FET de 486.

Los principales tipos de trabajos (categorías impuestas por la base de datos) fueron: revisiones narrativas (15\%), revisiones sistemáticas (8\%), ensayos clínicos aleatorizados (8\%), casos y controles (8\%), estudios observacionales (7\%), ensayos clínicos no aleatorizados (5\%), metaanálisis (4\%). No se identificaron estudios multicéntricos.

Los países más productivos fueron: Estados Unidos (21,10\%), India (12,84\%), Brasil (6,42\%), Finlandia (5,50\%), Japón (5,04\%) y Suecia (4,59\%).

\section{Discusión}

Las primeras publicaciones sobre biomarcadores salivales que registró DOSS, fue durante 2004, de ahí la elección del año de inicio de este estudio. Coincide con 2 iniciativas históricas emitidas por el Instituto Nacional de Investigación Dental y Craneofacial (NIDCR) referidas a descifrar el proteoma salival humano y desarrollar dispositivos que puedan analizar proteínas para aplicaciones de medicina de precisión personalizadas (18). 
En este estudio, la cantidad de publicaciones mundiales sobre biomarcadores salivales ha aumentado desde 2004 a 2018, en coincidencia con la posibilidad de usar la saliva como líquido de diagnóstico de enfermedades orales y sistémicas. (5)

Asimismo, el avance de técnicas de descubrimiento de nuevos biomarcadores también fue una razón potencial indirecta detrás del creciente número de publicaciones vistas en los últimos años (2)

Como lo afirma Price de Solla (19), una literatura puede ser linear (autores precursores), luego transformarse en exponencial para alcanzar su punto de saturación con una forma logística. Este no parece ser el caso de la literatura sobre biomarcadores salivales que, pasados 61 años desde sus publicaciones iniciales, aún sigue en permanente crecimiento. El primer intento de diagnóstico con proteína salival fue realizado por Hoerman (1959), quien demostró que los pacientes con cáncer de próstata exhibieron elevada actividad enzimática de fosfatasa acida en la saliva parotídea (18).

Estados Unidos presentó el mayor número de publicaciones, en concordancia con otros estudios relevantes sobre enfermedades orales $(20,21)$. El gran número de científicos del país, con el apoyo de instituciones oficiales (Instituto Nacional de Salud-USA), han realizado estudios de la saliva durante mucho tiempo, con hallazgos que crearon una base sólida para investigaciones posteriores (22).

En este trabajo, entre los países más productivos se observan varios europeos y asiáticos. El crecimiento en el número de publicaciones en países emergentes refleja los esfuerzos realizados para lograr presencia en el entorno internacional (23).

Entre los países productivos se halló también un país de América del Sur, Brasil. Presentó un importante número de publicaciones, en concordancia con registros internacionales $(24,25)$. Posiblemente, el establecimiento de redes de investigación creó nuevas oportunidades de cooperación, aumentó la producción científica y transferencia de tecnología, facilitando así la colaboración internacional en los manuscritos (26).

Los resultados de este estudio revelaron que, predominó la autoría única. La distribución porcentual de autores no se ajustó a la ley de Lokta, que establece un 60\% para autores con solo un artículo, 15\% para dos artículos y solo el seis por ciento para más de 10 artículos (27). Esta distribución confirma que biomarcadores salivales es un tema en desarrollo, con un estudio intensivo reciente.

En este trabajo, el número promedio de autores por publicación creció en los últimos años. Este aumento en la coautoría se repitió en otros campos de las ciencias de la salud $(28,29)$. Puede explicarse por la creciente necesidad de investigación interdisciplinaria, que a veces es una condición impuesta por las fuentes de financiamiento (10). Además, estas cifras de coautoría, indicarían un desarrollo 
bibliométrico internacional en dirección a la especialización del tema biomarcadores salivales. Cuando la comunidad académica, comprometida con un tema específico presenta un comportamiento de colaboración frecuente, logra mayor impacto en las investigaciones (30).

En la odontología basada en la evidencia científica, la toma de decisiones clínicas se realiza en base al diseño metodológico de los estudios. Metaanálisis, revisiones sistemáticas y ensayos clínicos aleatorizados presentan un alto nivel de evidencia. Los estudios de nivel intermedio y bajo son ensayos no aleatorizados, estudios de cohortes, estudios de casos y controles, estudios descriptivos (31). La mayoría de los trabajos, en relación con biomarcadores salivales, publicados en revistas indizadas en DOSS corresponden a los niveles intermedio y bajo de evidencia científica. Los ensayos clínicos aleatorios constituyen el mejor diseño de estudio para determinar la efectividad de las intervenciones, pero los estudios observacionales (descriptivos y analíticos) y los ensayos clínicos no aleatorizados, también ofrecen información válida (23).

En este estudio, la ausencia de estudios multicéntricos no fue lo esperado, dada la enorme carga de los problemas de salud mundiales relacionados con los biomarcadores salivales. Las colaboraciones multicéntricas realizadas de forma cooperativa, tienen ventajas importantes como reclutar más pacientes en un plazo mucho más corto y permitir una mayor generalización de los resultados $(32,33)$.

Los estudios bibliométricos revelan que las publicaciones científicas dentales, en la década del 2000 y la actual, son de mayor calidad, un reflejo de la creciente colaboración entre los grupos de investigación (34). Asimismo, a través de los años se han publicado más investigaciones originales en odontología en comparación con las revisiones (35), afirmación corroborada en este estudio a través de DOSS.

Coincidiendo con otro trabajo (10), las redes de coautoría identificadas en el presente estudio muestran que los autores más productivos y con mayor FET colaboran con más frecuencia con los autores que pertenecen a la misma organización y/o país.

En este estudio, las redes principales de colaboración identificadas fueron de países europeos y americanos. La colaboración fue mayor entre los países del mismo continente, aunque se identificó una tendencia hacia una mayor colaboración entre Ios países europeos y USA, en conjunto con países emergentes como India y Brasil. Este patrón de colaboración internacional, con fuertes predominios regionales, involucrando sólo unos pocos autores, dominado por Estados Unidos y algunos países europeos, es una constante en todas las publicaciones científicas (36). 
Los síntomas del cáncer a menudo no son específicos y están ausentes, hasta que los tumores ya han hecho metástasis. Por lo tanto, existe una demanda urgente para desarrollar herramientas rápidas, altamente precisas y no invasivas para el cribado del cáncer (37). Este trabajo mostró que $20 \%$ de las publicaciones sobre biomarcadores publicados en revistas indizadas en DOSS, informaron las posibilidades que puede tener la saliva como fluido diagnóstico de carcinomas orales $(38,39)$.

En ese mismo sentido, un $37 \%$ de las publicaciones estuvieron asociadas con la enfermedad periodontal. Todas coincidían, en la necesidad de un diagnóstico y monitoreo temprano mediante biomarcadores específicos, para resistir la progresión de la enfermedad y evitar la pérdida dentaria $(40,41)$.

\section{Conclusiones}

Basado en las crecientes inquietudes sobre biomarcadores salivales, este trabajo demostró un crecimiento de la documentación. Sin embargo, la ausencia de estudios multicéntricos manifiesta la necesidad de mayores acciones de la comunidad científica internacional para integrarse en el estudio del tema.

En este sentido, el presente trabajo ha generado una línea de investigación documental que servirá como insumo de base para la gestión de políticas públicas, específicamente la posibilidad de implementar el diagnóstico de enfermedades a través de los analitos de la saliva y estimular los estudios multicéntricos sobre biomarcadores salivales.

\section{Conflicto de intereses}

El autor declara que no existe ningún conflicto de interés

\section{Limitaciones}

Los límites de esta investigación, están dados por la cobertura documental de la base de datos, que no registran todas las publicaciones científicas internacionales biomarcadores salivales, siendo la cantidad de revistas indizadas, los factores que relativizan este estudio.

Para minimizar los posibles defectos que son inherentes a la metodología bibliométrica, se utilizó una base de datos adecuada a la temática, con más cobertura en odontología. La estrategia de búsqueda implementada fue exhaustiva y válida. 


\section{Referencias}

1. Dawes C, Pedersen AM, Villa A, Ekström J, Proctor GB, Vissink A, et al. The functions of human saliva: A review sponsored by the World Workshop on Oral Medicine VI. Arch Oral Biol. 2015;60(6):863-74. DOI: 10.1016/j.archoralbio.2015.03.004

2. Castagnola M, Scarano E, Passali GC, Messana I, Cabras T, lavarone F, et al. Salivary biomarkers and proteomics: future diagnostic and clinical utilities. Acta Otorhinolaryngol Ital. 2017;37(2):94-101. DOI: 10.14639/0392-100X-1598

3. Yoshizawa JM, Schafer CA, Schafer JJ, Farrell JJ, Paster BJ, Wong DT. Salivary biomarkers: toward future clinical and diagnostic utilities. Clin Microbiol Rev. 2013;26(4):781-91. DOI: 10.1128/CMR.00021-13

4. Spielmann N, Wong DT. Saliva: diagnostics and therapeutic perspectives. Oral Dis. 2011;17(4):345-54. DOI: 10.1111/j.1601-0825.2010.01773.x.

5. Javaid MA, Ahmed AS, Durand R, Tran SD. Saliva as a diagnostic tool for oral and systemic diseases. J Oral Biol Craniofac Res. 2016;6(1):66-75. DOI: 10.1016/j.jobcr.2015.08.006.

6. Shah S. Salivaomics: The current scenario. J Oral Maxillofac Pathol. 2018;22(3):375-381. DOI: 10.4103/jomfp.JOMFP_171_18

7. Pu QH, Lyu QJ, Su HY. Bibliometric analysis of scientific publications in transplantation journals from Mainland China, Japan, South Korea and Taiwan between 2006 and 2015. BMJ Open. 2016;6(8):e011623. DOI: 10.1136/bmjopen-2016-011623

8. Nasli-Esfahani E, Farzadfar F, Kouhnavard M, Ghodssi-Ghassemabadi R, Khajavi A, Peimani M, et al. Iran diabetes research roadmap (IDRR) study: a preliminary study on diabetes research in the world and Iran. J Diabetes Metab Disord. 2017;16:9. DOI: 10.1186/s40200-017-0291-9

9. Juárez RP, Barrere RM. Estudio Bibliométrico de la Producción Científica Argentina en Cáncer a través de las Bases de Datos Medline y Lilacs. Rev Salud Pública (Córdoba). 2017; 21(2):3142. DOI: $10.31052 / 1853.1180$

10. Tarazona B, Vidal IA, Alonso AA. Bibliometric analysis of the scientific production in implantology (2009-2013). Clin Oral Implants Res. 2017;28(7):864-870. DOI: 10.1111/clr.12891 
Análisis bibliométrico de la producción científica internacional sobre biomarcadores salivales durante el período 2004-2018

11. Jaimes-Monroy G, Valderrama-Salgado Al, Prieto-Cárdenas ES, Rincón-Bermúdez CM. Scientific production on dental caries 2014-2018: a bibliometric study in Web of Science. Rev Fac Odontol Univ Antioq. 2019; 31(1-2): 77-90. DOI: http://dx.doi.org/10.17533/udea.rfo. v31n1-2a7

12. Tarazona-Alvarez B, Lucas-Dominguez R, Paredes-Gallardo V, Alonso-Arroyo A, Vidal-Infer A. A bibliometric analysis of scientific production in the field of lingual orthodontics. Head Face Med. 2019;15(1):23. DOI: 10.1186/s13005-019-0207-7

13. Zhang W, Yuan H. A Bibliometric Analysis of Energy Performance Contracting Research from 2008 to 2018. Sustainability. 2019;11(13):3548. DOI: 10.3390/su11133548

14. Wang Y, Wang Q, Zhu R, Yang C, Chen Z, Bai Y, et al. Trends of spinal tuberculosis research (1994-2015): a bibliometric study. Medicine. 2016;95(38):e4923. DOI: 10.1097/MD.000000 0000004923

15. Song J, Zhang H, Dong $\mathrm{H}$. A review of emerging trends in global PPP research, analysis and visualization. Scienomtrics. 2016;107(3):1111-1147. DOI: 10.1007/s11192-016-1918-1

16. Moodley J, Singh V, Kagina BM, Abdullahi L, Hussey GD. A bibliometric analysis of cancer research in South Africa: study protocol. BMJ Open. 2015;5(2):e006913. DOI: 10.1136/ bmjopen-2014-006913

17. Sweileh WM. Bibliometric analysis of peer-reviewed literature in transgender health (1900 2017). BMC Int Health Hum Rights. 2018;18(1):16. DOI: 10.1186/s12914-018-0155-5

18. Dawes C, Wong DTW. Role of Saliva and Salivary Diagnostics in the Advancement of Oral Health. J Dent Res. 2019;98(2):133-141. DOI: 10.1177/0022034518816961

19. Price, JD de Solla. Diseases of science, en Science Since Babylon, ed. ampliada, New Haven y London: Yale University Press;1975. p. 161-195.

20. Pena-Cristóbal M, Diniz-Freitas M, Monteiro L, Diz Dios P, Warnakulasuriya S. The 100 most cited articles on oral cancer. J Oral Pathol Med. 2018;47(4):333-344. DOI: 10.1111/jop.12686

21. Foy JP, Bertolus C, Goudot P, Deneuve S, Blanc E, Lasset C, et al. Bibliometric analysis of a century of research on oral erythroplakia and leukoplakia. J Oral Pathol Med. 2018;47(4):388395. DOI: $10.1111 /$ jop.12683 
22. Ai J, Smith B, Wong DT. Saliva Ontology: An ontology-based framework for a Salivaomics Knowledge Base. BMC Bioinformatics. 2010;11:302. DOI: 10.1186/1471-2105-11-302

23. Kramer PF, Onetto J, Flores MT, Borges TS, Feldens CA. Traumatic Dental Injuries in the primary dentition: a 15-year bibliometric analysis of Dental Traumatology. Dent Traumatol. 2016;32(5):341-346. DOI: 10.1111/edt.12262

24. Scimago Journal \& Country Rank. Country Ranking. [Internet]. España: Scimago Lab; 2020 [consultado 13 de enero 2020]. Accesible en: http://www.scimagojr.com

25. Red de Indicadores de Ciencia y Tecnología. Iberoamericana e Interamericana. [Internet]. Indicadores comparativos bibliométricos. Buenos Aires: RICYT; 2020 [consultado 13 de enero 2020]. Accesible en: http://www.ricyt.org/2010/09/comparativos/

26. Shaw IF. Research networking and collaboration: a case study. Qual Inq. 2018;25(9-10): 11281136. DOI: 10.1177/1077800418809144

27. Lotka AJ. The frequency distribution of scientific productivity. J Wash Acad Sci. 1926;16(12):317-323.

28. Baghaei Lakeh A, Ghaffarzadegan N. Global Trends and Regional Variations in Studies of HIV/ AIDS. Sci Rep. 2017;7:4170. DOI: 10.1038/s41598-017-04527-6

29. Helgesson, G., Eriksson, S. Responsibility for scientific misconduct in collaborative papers. Med Health Care and Philos. 2018;21(3):423-430. DOI:10.1007/s11019-017-9817-7

30. Gazni A, Didegah F. Investigating different types of research collaboration and citation impact: A case study of harvard university publications. Scientometrics. 2011;87(2):251-265. DOI:10.1007/s11192-011-0343-8

31. Dhar V. Evidence-based dentistry: An overview. Contemp Clin Dent. 2016;7(3):293-4. DOI: 10.4103/0976-237X.188539

32. Irving SY, Curley MA. Challenges to conducting multicenter clinical research: ten points to consider. AACN Adv Crit Care. 2008;19:164-9. DOI: 10.1097/01.AACN.0000318119.67061.0f

33. Sprague S, Matta JM, Bhandari M. Multicenter Collaboration in Observational Research: Improving Generalizability and Efficiency. J Bone Joint Surg Am. 2009;91 (Suppl 3):80-6. DOI: 10.2106/JBJS.H.01623 
Análisis bibliométrico de la producción científica internacional sobre biomarcadores salivales durante el período 2004-2018

34. Jayaratne YS, Zwahlen RA. The evolution of dental journals from 2003 to 2012: a bibliometric analysis. PLoS One. 2015;17:e0119503. DOI: 10.1371/journal.pone.0119503

35. Kanavakis G, Dombroski MM, Malouf DP, Athanasiou AE. Demographic characteristics of systematic reviews, meta-analyses, and randomized controlled trials in orthodontic journals with impact factor. Eur J Orthod. 2016;38(1):57-65. DOl: 10.1093/ejo/cjv012

36. Knowledge, networks and nations: Global scientific collaboration in the 21st century. [Internet]. London: The Royal Society;2011 [consultado 19 de enero 2020]. Accesible en: https://royalsociety.org/topics-policy/projects/knowledge-networks-nations/report/

37. Wang X, Kaczor-Urbanowicz KE, Wong DT. Salivary biomarkers in cancer detection. Med Oncol. 2017;34(1):7. DOI: 10.1007/s12032-016-0863-4

38. Guerra ENS, Acevedo AC, Leite AF, Gozal D, Chardin H, De Luca Canto, G. Diagnostic capability of salivary biomarkers in the assessment of head and neck cancer: A systematic review and meta-analysis. Oral Oncol. 2015; 51(9): 805-818. DOI: 10.1016/j.oraloncology.2015.06.010

39. Kaur J, Jacobs R, Huang Y, Salvo N, Politis C. Salivary biomarkers for oral cancer and pre-cancer screening: a review. Clin. Oral Investig. 2018;22(2):633-640. DOI: 10.1007/s00784-018-2337-x

40. Ghallab NA. Diagnostic potential and future directions of biomarkers in gingival crevicular fluid and saliva of periodontal diseases: Review of the current evidence. Arch Oral Biol. 2018;87:115-124. DOI: 10.1016/j.archoralbio.2017.12.022

41. Zinzuvadia K, Zohrehei H, Dabeshlim K, Kanasi E, Grover S, Jones JA. Salivary Biomarkers in Periodontal Diseases - a Systematic Review. Int. J. Med. Dent [Internet]. 2018 [cited 2020 Feb 2];22(3): 263-279. Available from: https://search.ebscohost.com/login.aspx?

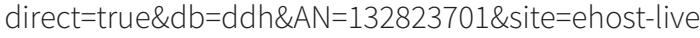

